\title{
Strong lensing by fermionic dark matter in galaxies
}

\author{
L. Gabriel Gómez, ${ }^{1,2,3}$ C. R. Argüelles, ${ }^{3,4}$ Volker Perlick, ${ }^{5}$ J. A. Rueda, ${ }^{1,3,6}$ R. Ruffini ${ }^{1,3,6}$ \\ ${ }^{1}$ Dipartimento di Fisica and ICRA, Sapienza Università di Roma, P.le Aldo Moro 5, I-00185 Rome, Italy \\ ${ }^{2}$ University of Nice-Sophia Antipolis, 28 Av. de Valrose, 06103 Nice Cedex 2, France \\ ${ }^{3}$ ICRANet, Piazza della Repubblica 10, I-65122 Pescara, Italy \\ ${ }^{4}$ Grupo de Astrofisica, Relatividad y Cosmología, Facultad de Ciencias Astronomicas y Geofisicas \\ Universidad Nacional de La Plata and CONICET, \\ Paseo del Bosque S/N 1900 La Plata, Pcia. de Buenos Aires, Argentina \\ ${ }^{5}$ ZARM, University of Bremen, 28359 Bremen, Germany and \\ ${ }^{6}$ ICRANet-Rio, CBPF, Rua Dr. Xavier Sigaud 150, Rio de Janeiro, RJ, 22290-180, Brazil
}

(Dated: October 11, 2016)

\begin{abstract}
It has been shown that a self-gravitating system of massive $\mathrm{keV}$ fermions in thermodynamic equilibrium correctly describes the dark matter (DM) distribution in galactic halos (from dwarf to spiral and elliptical galaxies) and that, at the same time, it predicts a denser quantum core towards the center of the configuration. Such a quantum core, for a fermion mass in the range of $50 \mathrm{kev} \lesssim m c^{2} \lesssim 345 \mathrm{keV}$, can be an alternative interpretation of the central compact object in Sgr $\mathrm{A}^{*}$, traditionally assumed to be a black hole $(\mathrm{BH})$. We present in this work the gravitational lensing properties of this novel DM configuration in nearby Milky Way-like spiral galaxies. We describe the lensing effects of the pure DM component both on halo scales, where we compare them to the effects of the Navarro-Frenk-White and the Non-Singular Isothermal Sphere DM models, and near the galaxy center, where we compare them with the effects of a Schwarzschild BH. For the particle mass leading to the most compact DM core, $m c^{2} \approx 10^{2} \mathrm{keV}$, we draw the following conclusions. At distances $r \gtrsim 20 \mathrm{pc}$ from the center of the lens the effect of the central object on the lensing properties is negligible. However, we show that measurements of the deflection angle produced by the DM distribution in the outer region at a few $\mathrm{kpc}$, together with rotation curve data, could help to discriminate between different DM models. In the inner regions $10^{-6} \mathrm{pc} \lesssim r \lesssim 20 \mathrm{pc}$, the lensing effects of a DM quantum core alternative to the BH scenario, becomes a theme of an analysis of unprecedented precision which is challenging for current technological developments. We show that at distances $\sim 10^{-4} \mathrm{pc}$ strong lensing effects, such as multiple images and Einstein rings, may occur. Large differences in the deflection angle produced by a DM central core and a central BH appear at distances $r \lesssim 10^{-6} \mathrm{pc}$; in this regime the weak-field formalism is no longer applicable and the exact general-relativistic formula has to be used for the deflection angle which may become bigger than $2 \pi$. An important difference in comparison to BHs is in the fact that quantum DM cores do not show a photon sphere; this implies that they do not cast a shadow (if they are transparent). Similar conclusions apply to the other DM distributions for other fermion masses in the above specified range and for other galaxy types.
\end{abstract}

Keywords: Dark matter: Fermions, halo- Galaxy: lens model, central compact object

\section{INTRODUCTION}

Most of the intriguing problems in particle physics and cosmology are related to the nature of the dark matter (DM) that composes approximately $80 \%$ of matter in the Universe [1]. In a self-consistent particle DM model aimed to understand the quasi relaxed DM halo structures, the underlying phase-space distribution and self-gravitation establishes how the DM distributes in the galaxy. Namely, the distribution of DM inside the galaxy (e.g. mass, density and pressure profiles) can be obtained from the solution of the hydrostatic equilibrium equations and the corresponding (phase-space dependent) equation of state (see e.g. [2]).

Before a DM halo enters in the steady state we observe, and due to the collisionless nature of the DM particles, specific relaxation mechanisms such as violent relaxation take place in a few dynamical times, giving rise to quasi-stationary states which can be described by (coarse grained) phase-space distributions of the Fermi-
Dirac type $[3,4]$. Indeed, it has been recently shown that a model of DM based on a self-gravitating system of fermions in thermodynamic equilibrium accurately describes the distribution of DM in galactic halos, when contrasted with observations [5-7]). In Ref. [5], it was shown that such a self-gravitating system of fermionic DM shows a general DM density distribution, hereafter the RAR profile, with a compact core - diluted halo structure (see Sec. II for details). More recently, following the more complex and realistic statistical approach accounting for escape of particles, in Ref. [7] it was introduced a cutoff in the fermion momentum distribution. Such a momentum cutoff serves to account for the finite size of galaxies, generalizing the previous RAR profile [5]. Both Refs. [5] and [7] have put constraints on the mass of these fermions, hereafter called inos, using known observational properties of galaxies such as the flatness of the rotation curves, the mass and radius of galaxies, as well as observationally-inferred correlations involving many different galaxy types. 
On the other hand, gravitational lensing (GL) has been widely used to determine the distribution of DM in galaxies and galaxy clusters [8, 9]. Hence, given a specific density profile it is systematically possible to infer the GL properties for any lens system or vice versa, i.e., if we know the lensing signal, we can reconstruct the mass distribution of the lens under some assumptions of it. Moreover, it was presented a Bayesian statistical method in [10] that permit to reconstruct a model independent mass profile without initial assumptions by combining measurements of magnification bias along with lens distortion. For instance, the gravitational lensing properties given by the phenomenological Navarro-Frenk-White (NFW) profile, commonly used to describe the cold dark matter (CDM) distribution of halos, have been very well investigated (see, e.g., Ref. [11], and references therein). The same applies to the non-singular isothermal sphere (NSIS) profile [12]. Interestingly, the lensing data are better fitted by the latter kind (see e.g. [13]), which is cored-like (i.e. with a shallower inner DM halo density profile in contrast to the more cuspy NFW one), in a way similar to the RAR profile in that galaxy region (see Fig. 3 in [5]). Particular attention has been paid in the last decade to single galaxies where strong lensing effects are commonly present. Surveys such as SWELLS $[14,15]$ and DiskMass [16], among others, have placed constraints on the properties of spiral galaxies, revealing for instance the DM fraction within 2.2 disk radii, the inner logarithmic slope of the DM halo profile, as well as the stellar mass component (disk plus bulge). Including kinematic analysis, it is also possible to break the disk-halo degeneracy [14] and to put a more stringent constraint on the aforementioned properties (see also [17] for a theoretical study and [18] for a summary of the DM properties). Likewise, the slope of the average DM projected density profile in the innermost regions of massive early type galaxies, has been constrained by using strong gravitational lensing data along with stellar dynamics and stellar population [19-21]. Recently, the DM density profile has been constrained at a few kpc, contributing significantly in the same way as the stellar component does [22, 23], exhibiting that these types of galaxies have a non-negligible amount of DM in their central regions.

Observations also indicate that most galaxies host a massive central compact object, usually assumed to be a black hole (BH). Its presence substantially affects lensing features such as the critical curves and the formation (suppression) of an additional (existing) faint central image, as predicted in [24] and already observed [25]. Remarkably, such effects depend on the halo core radius and a critical value of the mass of the $\mathrm{BH}[22,24]$, leading to a strong degeneracy between these parameters.

At this point we turn back to the discussion of DM models on galaxy scales to recall an interesting feature of the RAR model [7]: its DM central core, hereafter DMCC, can be compact enough to correctly describe the observational properties in the Galactic center, which are usually associated with the existence of a massive $\mathrm{BH}$ centered in Sgr A*. Namely, it can produce a gravitational potential which suffices to explain the dynamics of the stars closest to Sgr A*, the S-cluster stars (see Ref. [7] for details). Thus, the RAR profile could, in principle, explain the MW properties from the center all the way to the halo.

It is thus natural to ask ourselves about the GL properties of nearby lensing galaxies modeled within the RAR model. Without loss of generality, we compute in this work the GL properties of DM halos for spiral type galaxies such as the MW. In addition to the inclusion of the halo part, which is slightly but appreciably distinguishable from other DM models, we consider the lensing effects of the DM distribution near the GC where a maximum deflection of light is predicted, in contrast to standard models of DM such as NFW and NSIS. We also show that, at such scales, the deflection angles are no longer small so that the exact equations from general relativity must be used.

This work is organized as follows: In Sec. II we describe the general features of the novel model of DM fermions and compare them with the ones obtained by the standard density profiles. We compute in Sec. III the gravitational lensing properties of our DM distribution in Milky Way-like galaxies and compare them in the halo with those of the NFW and NSIS profiles and in the core with those of a Schwarzschild BH as they have been predicted for Sgr A* [26]. Finally we present a general discussion in Sec. IV of the GL properties of the fermionic DM distribution in galaxies.

\section{THE DENSITY PROFILES}

We first recall the widely used phenomenological DM density profile arising within the $\Lambda \mathrm{CDM}$ cosmological paradigm, i.e. the NFW profile [27]

$$
\rho(r)=\frac{\rho_{c}}{\left(r / r_{s}\right)\left(1+r / r_{s}\right)^{2}},
$$

where $\rho_{c}$ is the characteristic density and $r_{s}$ is the scale radius. This density profile exhibits a sharp cusp in the inner region $\rho \propto r^{-1}$ while in the halo part the density scales as $\rho \propto r^{-3}$.

Another often adopted DM density profile which also yields the asymptotic flatness of the rotation curves is represented by the non-singular isothermal (NSIS) profile [28]:

$$
\rho(r)=\frac{\rho_{0}}{1+\left(r / r_{0}\right)^{2}},
$$

where $\rho_{0}$ is the central density and $r_{0}$ is the core radius.

We turn now to the RAR profile $[5,7]$ which describes the DM distribution along the entire galaxy in a continuous way, i.e. from the halo part to the GC and without spoiling the baryonic component which dominates at intermediate scales (see [7] for details). 
Assuming a self-gravitating system of massive fermions (within the standard Fermi-Dirac phase-space distribution) in thermodynamic equilibrium, the DM density profile was computed in [5]. By imposing fixed boundary conditions at the halo and including the fulfillment of the rotation curves data, the parameters of the system have been constrained. This procedure was applied for different types of galaxies from dwarfs to big spirals exhibiting a universal compact core - diluted halo density profile. An extended version of the RAR model was recently presented [7], by introducing a fermion energy cutoff $\epsilon_{c}$ in the fermion distribution. This is also motivated by the formal stationary solution (Fermi-Dirac-like) of the generalized statistics which includes the effects of escape of particles and violent relaxation [4]. The new emerging density profile serves to account for the finite galaxy sizes due to the more realistic boundary conditions, while it opens the possibility to achieve a more compact solution for the quantum core working as a good alternative to the BH scenario in Sgr A* (see Fig. 1).

Motivated by these features between the DM profiles, we compute in the next section both the GL properties of MW-type galaxies for the RAR model and the GL effect of the DMCC in order to study the possibility of strong lensing effects around the GC. We describe now the RAR profile following Ref. [7]. by

The density $\rho$ and pressure $P$ for this system are given

$$
\begin{aligned}
\rho & =\frac{g}{h^{3}} m \int_{0}^{\epsilon_{c}} f_{c}(p)\left(1+\frac{\epsilon(p)}{m c^{2}}\right) d^{3} p, \\
P & =\frac{2}{3} \frac{g}{h^{3}} \int_{0}^{\epsilon_{c}} f_{c}(p) \frac{1+\epsilon(p) / 2 m c^{2}}{1+\epsilon(p) / m c^{2}} d^{3} p
\end{aligned}
$$

where $g$ denotes as usual the particle spin degeneracy and $f_{c}(p)$ is the phase space distribution function including an energy cutoff:

$$
f_{c}(p)= \begin{cases}\frac{1-e^{\left(\epsilon-\epsilon_{c}\right) / k T}}{e^{(\epsilon-\mu) / k T}+1} & \epsilon \leqslant \epsilon_{c} \\ 0 & \epsilon>\epsilon_{c} .\end{cases}
$$

Here $\epsilon=\sqrt{c^{2} p^{2}+m^{2} c^{4}}-m c^{2}$ is the particle kinetic energy, $m$ is the particle mass, $\mu$ is the chemical potential (with the particle rest mass subtracted off), $T$ is the temperature, $k$ is the Boltzmann constant, and $h$ is the Planck constant.

Considering the spherically symmetric space-time described by the metric

$$
d s^{2}=e^{\nu} d t^{2}-e^{\lambda} d r^{2}-r^{2} d \Theta^{2}-r^{2} \sin ^{2} \Theta d \phi^{2},
$$

along with the thermodynamic equilibrium conditions $e^{\nu / 2} T=$ const. and $e^{\nu / 2}\left(\mu+m c^{2}\right)=$ const. and the equation of state given by Eqs. (3) and (4), the dimensionless Einstein equations are finally obtained (see Ref. [7], for details)

$$
\begin{aligned}
& \frac{d \hat{M}}{d \hat{r}}=4 \pi \hat{r}^{2} \hat{\rho}, \\
& \frac{d \theta}{d \hat{r}}=-\frac{\left[1-\beta_{0}\left(\theta-\theta_{0}\right)\right]}{\beta_{0}} \frac{\hat{M}+4 \pi \hat{P} \hat{r}^{3}}{\hat{r}^{2}(1-2 \hat{M} / \hat{r})}, \\
& \frac{d \nu}{d \hat{r}}=2 \frac{\hat{M}+4 \pi \hat{P} \hat{r}^{3}}{\hat{r}^{2}(1-2 \hat{M} / \hat{r})}, \\
& \beta(r)=\beta_{0} e^{\frac{\nu_{0}-\nu(r)}{2}} \\
& W(r)=W_{0}+\theta(r)-\theta_{0},
\end{aligned}
$$

where we have introduced the temperature parameter $\beta=k T / m c^{2}$, the degeneracy parameter $\theta=\mu / k T$ and the cutoff parameter $W=\epsilon_{c} / k T$. In addition, there have been introduced the dimensionless quantities $\hat{r}=r / \chi$, $\hat{M}=G M /\left(c^{2} \chi\right), \hat{\rho}=G \chi^{2} \rho / c^{2}$ and $\hat{P}=G \chi^{2} P / c^{4}$ with $\chi=2 \pi^{3 / 2}(\hbar / m c)\left(m_{p} / m\right)$ and $m_{p}$ being the Planck mass. The subscript 0 in the Einstein equations denotes the initial condition values. Thus, the fermionic DM density profile was computed numerically and characterized by a quantum central core of almost constant density, an intermediate transition region followed by an extended plateau and a Boltzmannian density tail $\rho \propto r^{-\alpha}$, with $\alpha>2$ due to the cutoff condition (see Fig. 1). This latter feature, together with the addition of the standard baryonic disk component, leads to the observed flat rotation curves (see [7] for the specific analysis of the MW). Based on this property, a family of solutions can be obtained to guarantee the fulfillment of both the boundary conditions of the halo part and rotation curve data, with different central quantum cores of different compactness presenting a clear dependence on the fermion mass (see Fig. 1 for the case of the three solutions of interest).

The size of the degenerate quantum core depends on the particle mass as can be seen in Fig. 1 for the case of the MW. As shown in [7], for an ino mass in the range of $48 \mathrm{keV} \lesssim m \lesssim 345 \mathrm{keV}$, the DM core has a size (and a mass) appropriate to describe the orbit of the S-cluster stars around Sgr A* [29]. Thus, the DM core represents a valid alternative to the central $\mathrm{BH}$ hypothesis. In addition, for such fermion masses the DM contribution properly reproduces the total rotation curve data, without spoiling the baryonic components available above parsec scale (see [7] for details and see [30] for the latest data available in the MW).

Therefore, the narrow particle mass range provides several solutions to satisfy either the rotation curve data in the halo part or both sets of data, namely including additionally the orbits of the S-cluster stars such as the S2 star, necessary to establish the compactness of the DMCC. A comparison between the RAR model, NFW profile and NSIS for MW-like spiral galaxies is also shown in Fig. 2, describing the outstanding inner structure below parsec scale for the inos profile. 


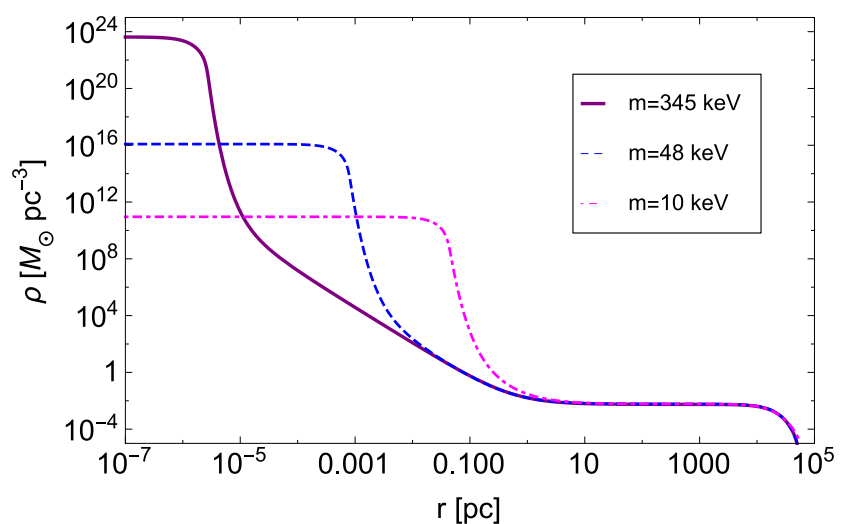

FIG. 1. Density profiles for MW-type galaxies for different particle mass values that satisfy the boundary conditions: $M_{D M}(r=20 k p c)=9 \times 10^{10} M_{\odot}$ (or equivalently $\left.M_{D M}(r=40 k p c)=2 \times 10^{11} M_{\odot}\right)$, with a DMCC of mass $M_{c}=4.2 \times 10^{6} M_{\odot}$. The set of initial conditions: $\theta_{0}=$ $32.2 ; \beta_{0}=2.7 \times 10^{-7} ; W_{0}=58.5, \theta_{0}=37.1 ; \beta_{0}=1.04 \times$ $10^{-5} ; W_{0}=65,20, \theta_{0}=45.8 ; \beta_{0}=4.0 \times 10^{-3} ; W_{0}=76.049$ for $m=10 \mathrm{keV}, m=48 \mathrm{keV}$ and $m=345 \mathrm{keV}$, respectively, have been here adopted from the original solutions computed in [7].

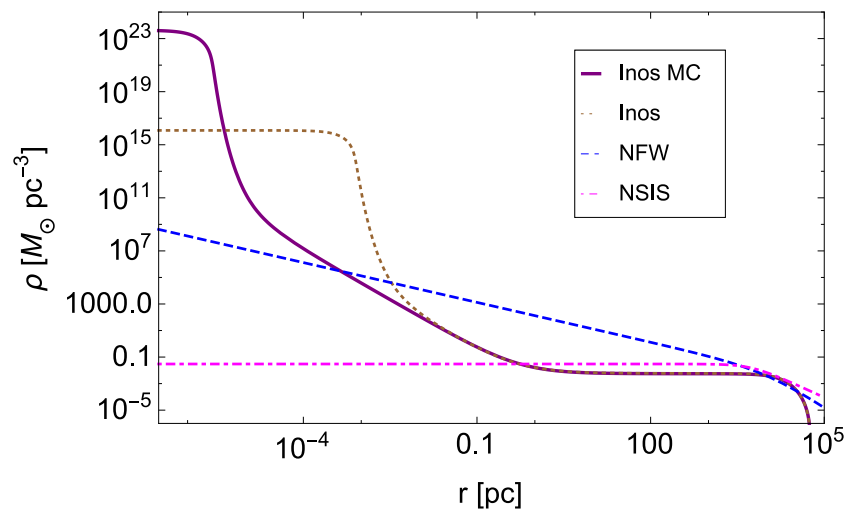

FIG. 2. Distribution of DM in MW-type galaxies predicted by the RAR model. The solid line, inos MC in the legend, refers to the most compact (MC) solution for $m=345 \mathrm{keV}$. For comparison, we show with the the dotted brown line, the solution for $m=48 \mathrm{keV}$ which we refer to as inos in the legend. There are also shown the NFW and NSIS profiles given by the formulas (1) and (2), respectively. The free parameters in these profiles were taken from [30] and [31], respectively, satisfying the same (total) rotation curve data as in the RAR case, with the corresponding considerations of bulge and disk counterparts.

\section{GRAVITATIONAL LENSING PROPERTIES}

As the RAR density profile cannot be given analytically, we here compute numerically the general GL properties for this model. The lensing effect causes the image of the source to be displaced, magnified (or demagnified), and sometimes splitted. Interestingly, these effects can be quantified and contrasted with observations; however, we do not focus on any particular lens system but rather on describing the GL properties of the RAR solutions in nearby MW-type spiral galaxies. To do so, we consider the particular solutions provided by the particle mass value $m=345 \mathrm{keV}$ and $m=48 \mathrm{keV}$ to account also for the central compact object. In subsections III A and III B we use the standard lensing formalism which is based on the assumption that the gravitational field is so weak that the deflection angles are small. In subsection III C we consider light rays that come so close to the central object that this approximation is no longer valid; there we have to calculate the deflection angles with the exact equations from general relativity. We also make a comparison of our results with the respective ones of the NFW and NSIS profiles as lensing models, in order to infer significant differences that might help us, along with rotation curve data, to discriminate between these DM galactic profiles. Henceforth, we will consider in all computations that the source and lens position are located at $z_{s}=2$ and $z_{l}=0.3$, respectively, which correspond to typical separations for both the source and the lens. The cosmological parameters have been taken from the last results of Planck [1]: $H=67.80 \mathrm{~km} \mathrm{~s}^{-1} \mathrm{Mpc}^{-1}$, $\Omega_{m}=0.3$ and $\Omega_{\Lambda}=0.7$ to determine the angular diameter distances of the system. For this cosmology, the scale in the lens plane is $1 \operatorname{arcsec}=4.74 \mathrm{kpc}$.

\section{A. Surface mass density and convergence}

Considering the lens system as an axially symmetric lens, the planar distribution of matter, i.e. the projected surface density, is obtained by integrating the threedimensional density profile $\rho(r)^{1}$ along the line of sight [32]

$$
\Sigma(\xi)=2 \int_{0}^{\infty} \rho(\xi, z) d z
$$

where $\xi$ is the impact parameter measured from the center of the lens. For this configuration, the mean surface density inside the radius $\xi$ is

$$
\bar{\Sigma}(\xi)=\frac{1}{\pi \xi^{2}} \int_{0}^{\xi} 2 \pi \xi^{\prime} \Sigma\left(\xi^{\prime}\right) d \xi^{\prime}
$$

A useful dimensionless quantity that characterizes the system is the convergence, defined as the ratio of the surface density and the critical density

$$
k(\xi)=\frac{\Sigma(\xi)}{\Sigma_{c r}},
$$

\footnotetext{
${ }^{1}$ Here the radial coordinate $r$ is related to cylindrical polar coordinates by $r=\sqrt{\xi^{2}+z^{2}}$.
} 
where $\Sigma_{c r}=\frac{c^{2}}{4 \pi G} \frac{D_{s}}{D_{l} D_{l s}}$ and $c$ is the speed of light. $D_{s}$, $D_{l}$ and $D_{l s}$ are the angular diameter distances of the observer to the source, of the observer to the lens, and of the lens to the source, respectively. Based on these definitions, we compute the convergence as a function of the impact parameter $\xi$ for all the above density profiles (see Fig. 3). It is common to give the impact parameter in units of a reference length $\xi_{0}$. For the RAR model, however, $\xi_{0}$ has not yet been identified, in contrast e.g. to the NFW profile. For this reason, all quantities related to lensing properties are plotted as a function of the physical impact parameter $\xi$ instead of the usually used dimensionless radius $\xi / \xi_{0}$. The influence of the DMCC of the RAR profile on the convergence can be clearly seen in Fig. 3. If $\kappa>1$, multiple images and Einstein rings may be formed. It is common to speak of "strong lensing" in such situations. Note, however, that we are still in the regime where the gravitational field is weak and bending angles are small. From the diagram we read that for the RAR profile strong lensing effects start to be notorious at a radius smaller than $10^{-4} \mathrm{pc}$, whereas in the halo part only weak lensing takes place, for all the density profiles, as expected [33].

The density of the RAR profile is, at small distances, several orders of magnitude higher than that of the NFW and NSIS counterparts. Hence, the compactness of the DM compact core may eventually lead to the additional formation of (relativistic) Einstein rings or (relativistic) multiple images, similarly to the case of a supermassive BH. This possibility will be analyzed in Sec. IIIC via a general relativity treatment beyond the weak-field approximation.

On the other hand, an interesting quantity that characterizes the system is the shear

$$
\gamma(\xi)=\frac{\bar{\Sigma}(\xi)-\Sigma(\xi)}{\Sigma_{c r}}
$$

which determines the distortion of images. For our DM fermionic configuration, the formation of peaks in the shear (which correspond to the formation of Einstein rings) appears presumably in zones where the surface density changes abruptly due to the dominance of DM (in the central and halo part). This feature is similar to that of the deflection angle plotted in Fig. 4.

\section{B. Deflection angle and magnification}

The deflection angle can be written as

$$
\hat{\alpha}(\xi)=\frac{4 G}{c^{2}} \frac{2 \pi \int_{0}^{\xi} \Sigma\left(\xi^{\prime}\right) \xi^{\prime} d \xi^{\prime}}{\xi}=\frac{4 G M(\xi)}{c^{2} \xi},
$$

with $M(\xi)$ being the mass enclosed by a circle of radius $\xi$. For any given density profile $\rho(r)$ we may (numerically) first calculate $\Sigma(\xi)$ and then $M(\xi)$ which, by (16), gives us the deflection angle $\hat{\alpha}(\xi)$. The results are shown for the NFW, NSIS and RAR profiles in Fig. 4 where the

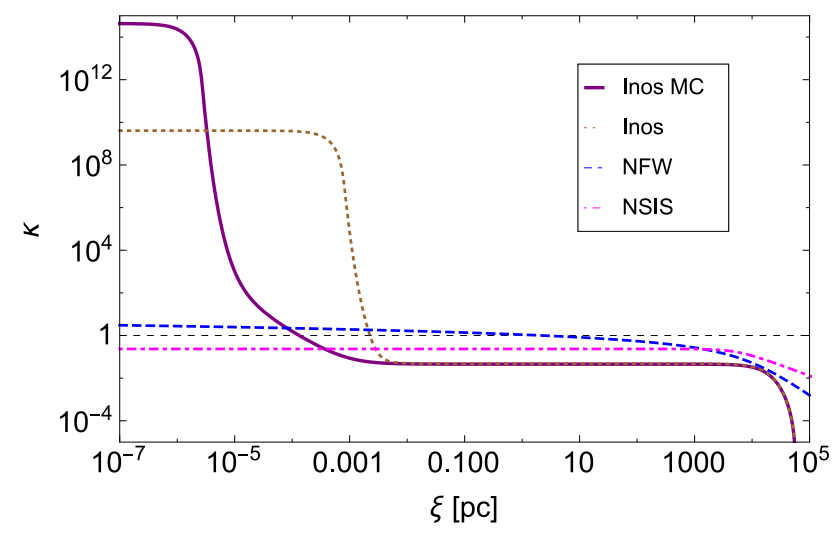

FIG. 3. Convergence for the NFW, NSIS and RAR density profiles. The condition of strong lensing is achieved for the RAR profile (inos MC) below $10^{-4} \mathrm{pc}$ while the halo part is characterized by weak lensing effects as well as for the other profiles.

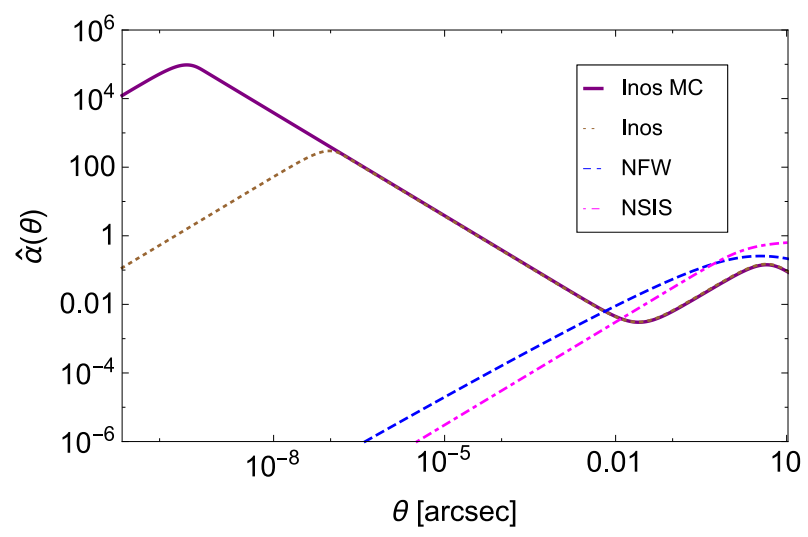

FIG. 4. Deflection angle for the NFW, NSIS and RAR density profiles. It can be seen the relation between the deflection angle and the rotation curve as it was found in [34]. See also Ref. [5] for the rotation curve behavior. The units for $\hat{\alpha}$ are given in arcsec.

deflection angle is plotted as a function of the position angle in the sky, $\theta=\xi / D_{l}$. For the RAR profile it can be observed that the deflection angle becomes larger when a light ray is close to the DMCC in comparison to one in the halo part, giving rise to one extra Einstein ring, see below. This is a unique feature of the GL produced by the RAR profile, since for the other profiles the maximum deflection angle is obtained in the halo part near the flat part of the rotation curve. The maximum deflection in the RAR profile case has the value $9.49 \times 10^{4} \operatorname{arcsec}(\hat{\alpha}=$ $0.46 \mathrm{rad})$, at radius 0.30 nano-arcsec $\left(1.42 \times 10^{-6} \mathrm{pc}\right)$ which is slightly underestimated because in this regime the weak-field approximation is actually no longer valid. An exact relativistic treatment will be given in the next section.

In the halo part, all the DM density profiles must fit as a first condition the rotation curve data. Moreover, a 
complementary requirement can be added by considering the light deflection by the galactic halo. With this extra information at disposal we can in principle discriminate between different halo models $[35,36]$ which predict slightly different deviations of light (of 0.1 arcsec) as can be seen in Fig. 4. On the other hand, starting from 2 mili-arcsec $(20 \mathrm{pc})$ the RAR profile produces a constantly increasing deflection in logarithmic scale, toward the central part, reaching the maximum value at 0.30 nano-arcsec $\left(1.42 \times 10^{-6} \mathrm{pc}\right)$ due to the DMCC gravitational potential (see Fig. 4).

Looking at the halo part, the deflecting angle (in arc$\mathrm{sec}$ ) for each profile is computed (according to the best fit parameters) at a distance $R^{G C}=8.3 \mathrm{kpc}$ (our distance from the galactic center) where the circular velocity is near its maximum value

$$
\begin{aligned}
\hat{\alpha}^{N F W}\left(R^{G C}\right) & \approx 0.26^{\prime \prime}, \\
\hat{\alpha}^{N S I S}\left(R^{G C}\right) & \approx 0.69^{\prime \prime}, \\
\hat{\alpha}^{i n o s}\left(R^{G C}\right) & \approx 0.15^{\prime \prime} .
\end{aligned}
$$

We recall that at such a radius scale the total circular velocity must fulfill $V_{c} \approx 220 \mathrm{~km} \mathrm{~s}^{-1}$, which implies that lensing data could serve as a discriminator between dark matter models. Interestingly, it has been recently inferred the model parameters, i.e. the total (disk+bulge) stellar mass, the DM halo asymptotic circular velocity, and the core radius among others, for the spiral galaxy lens SDSS J2141000 system by using either strong lensing data, kinematics data, or both combined [14]. This analysis shows that the uncertainty associated with the circular velocity when the optical emission and absorption line spectroscopy is considered only, is commonly less than that of strong lensing data (see table 5 in [14] for comparison). It implies directly that we can fit very well data from strong lensing since the RAR profile fits the rotation curves with a good precision [7].

In addition, the source is also magnified by a factor

$$
\mu(\theta)=\frac{1}{(1-k(\theta))^{2}-\gamma(\theta)^{2}} .
$$

To first order, the magnification depends on the convergence only. Negative values of $\mu$ correspond to inverted images and for large values of $\theta, \mu \rightarrow 1$ and the source is weakly affected by the lensing potential, while for $\theta=\theta_{E}$, the magnification diverges, corresponding to the formation of an Einstein ring. We calculate the magnification for all profiles and note the emergence of one extra Einstein ring due to the DMCC in addition to the halo part. The angular position of such an Einstein ring depends strongly on the compactness of the DMCC subject to the particle mass value between $48 \mathrm{kev} \lesssim m c^{2} \lesssim 345 \mathrm{keV}$. As we can see, the lensing signal is highly demagnified and its effect is indeed comparable to that produced by a $\mathrm{SMBH}$. This remarkable result is plotted in Fig. 5 which clearly shows the effect of the compact DM core near $10^{-7} \mathrm{pc}$ for the more compact solution. This result is also in agreement with the expected demagnified central

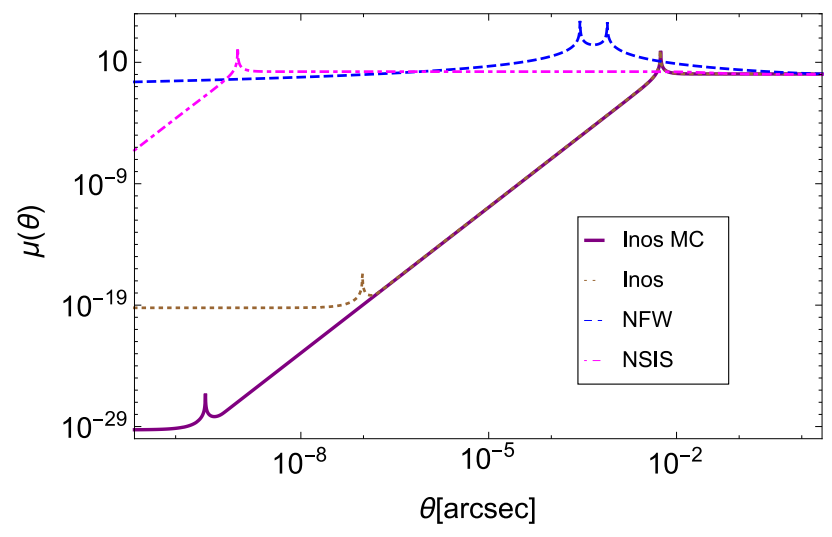

FIG. 5. Magnification factor for all the profiles listed in the legend and computed by Eq. (20).

image, since the central image flux depends inversely on the square of the surface density whereby concentrated density profiles should cause central images to be very faint $[22,24,25]$. We stress that Eq. (20) is only valid in the weak field limit, hence a general description must be used to account for the fully relativistic effects in the more compact solution, i.e, the one derived in the regime of large bending angles. Nevertheless, computing the magnification and the angular position of the additional Einstein ring in the full description of $\mathrm{GR}^{2}$, provides a value of $\theta_{E}=0.36$ nano-arcsec, which is only $18 \%$ above the one calculated within the weak field limit approximation, i.e. $\theta_{E}=0.30$ nano-arcsec.

It is important to note that we have used in all the calculations the standard lensing formalism, based on the assumptions that the gravitational field is weak and that the deflection angle is small. With this, it is possible to describe the properties of our fermionic DM gravitational lens system, which is expected to account for such effects very well in the halo part ${ }^{3}$. However, below $10^{-5} \mathrm{pc}$ strong bending must be taken into account to predict properly the deflection angle due to the DM distribution in that region. This will significantly affect the lensing properties of the DMCC. In the next section we calculate these lensing properties and compare them to those of a black hole.

\section{The regime of large bending angles: dark matter central core versus massive black hole}

In this part we study lensing due to a fermionic DM core in a galactic center when a light ray approaches it

\footnotetext{
2 See for instance section II in [26], where is described the exact equations for computing the magnification of images in this regime.

3 or at least far enough away from the DMCC to avoid strong bending effects
} 
very closely. For a compact object the bending angle may become large, even exceeding several multiples of $2 \pi$ meaning that the light ray makes several turns around the center. Therefore we have to use the full formalism of general relativity, beyond the weak-field approximation. Images associated with light rays that make at least one full turn around the center are often called "relativistic images" although "higher-order images" would be a better nomenclature. We will compare, in the regime where such images occur, the lensing features of a fermionic DM core with those of a black hole. The latter have been studied in great detail in the Schwarzschild BH scenario for Sgr A* [26], where relativistic images and relativistic Einstein rings are formed. Hence, a natural question that arises here is whether the DMCC compactness is large enough to account for the formation of such relativistic images. Hence, we attempt to answer this question by computing the deflection angle of light rays passing very closely by the DMCC and even inside of it since it is treated as transparent. To do so, we use the formula derived from the static spherically symmetric metric as a function of the closest light ray distance of approach $r_{0}$ $[37]$

$$
\hat{\alpha}\left(r_{0}\right)=2 \int_{r_{0}}^{\infty} \frac{e^{\lambda / 2} d r}{\sqrt{\left(r^{4} / b^{2}\right) e^{-\nu}-r^{2}}}-\pi
$$

where $b$ is the impact parameter:

$$
b=r_{0} \exp \left[-\nu\left(r_{0} / 2\right)\right] .
$$

In the case of the central BH hypothesis (as analyzed in $[26]$ ), the metric outside is described by the Schwarzschild solution

$$
\begin{gathered}
e^{\nu(r)}=1-\frac{2 G M}{c^{2} r} \\
e^{\lambda(r)}=\left(1-\frac{2 G M}{c^{2} r}\right)^{-1},
\end{gathered}
$$

where $M=4.2 \times 10^{6} M_{\odot}$ is the $\mathrm{BH}$ mass ${ }^{4}$. Instead, the metric coefficients for the fermionic model we are interested in here are obtained by solving the system of equations Eqs. (7)-(11) along with the equations for the density and the pressure, Eq. (3) and Eq. (4). The result for the deflection angle is plotted in Fig. 6 where we can see that the deflection caused by the DM central core is small in comparison to that of a black hole, although considerably beyond the validity of the weak-field formalism which assumes that $\hat{\alpha}$ may be identified with tan $\hat{\alpha}$. The maximum deflection, $\hat{\alpha} \approx 0.62$ (distinct from that obtained by the weak-field approach $\hat{\alpha}=0.46)$ is achieved at $r_{0} \approx 7.18 G M / c^{2}$ inside the DMCC. Not surprisingly, a

\footnotetext{
${ }^{4}$ For this mass, the Schwarzschild radius, $r_{s}=4.019 \times 10^{-7} \mathrm{pc}$.
}

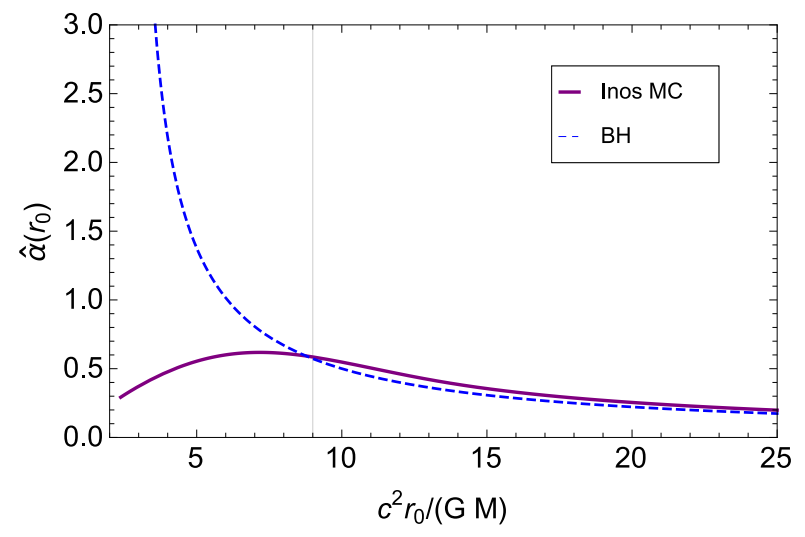

FIG. 6. Comparison between BH and fermionic DM quantum core of the inos $\mathrm{MC}$ configuration. The vertical line indicates the core radius of the DMCC: $r_{c} \approx 9 G M / c^{2}$. The deflections are given in radians.

similar feature was observed as in the case of other compact objects such as fully degenerate fermion stars as well as boson stars [38, 39] (see also Ref. [40] and references therein for a general discussion of compact objects and their gravitational lensing effects). Interestingly, still at distances larger than $r_{c}$, the deflection angles are appreciably different from the $\mathrm{BH}$ ones due to the contribution of the DM distribution leading to a slight difference in its gravitational potential. This is illustrated in Fig. 7.

The deflection angle for the DMCC can be computed approximately by the Einstein deflection angle provided $r_{0}$ is large, i.e, in the weak field limit ${ }^{5}$

$$
\hat{\alpha}\left(r_{0}\right)=\frac{4 G M}{c^{2} r_{0}}+\mathcal{O}\left(\frac{G^{2} M^{2}}{c^{4} r_{0}^{2}}\right),
$$

which at second order implies the value $\hat{\alpha}\left(r_{0}\right) \approx 0.18$ for $r_{0}=25 G M / c^{2}$, as can be also seen from Fig. 6. Hence Eq. (25) underestimates just a few percent level the deflection angle compared to that given for the full description Eq. (21) at $r_{0}=25 G M / c^{2}$. Below this radial scale Eq. (25) is no longer valid since it exceeds in more than $10 \%$ the correct relativistic result given by Eq. (21).

We recall that, for a Schwarzschild $\mathrm{BH}$, relativistic images are formed due to large bending of light near the photon sphere at $r=3 G M / c^{2}$. In this scenario, for the closest distance of approach $r_{0}=3.21 G M / c^{2}$ the deflection angle takes the value $\hat{\alpha}\left(r_{0}\right) \approx 3 \pi / 2$ which gives rise to the first relativistic Einstein ring. This somewhat gives a rough estimate of the compactness of the lens. By analogy, the compactness of the DMCC can be obtained as $c^{2} r_{c} /(G M) \approx 9$. However, the light rays can

\footnotetext{
5 It is important to note that this formula is strictly valid only for the Schwarzschild metric. However, at large radius values the fermionic solution tends to match the exterior Schwarzschild one.
} 


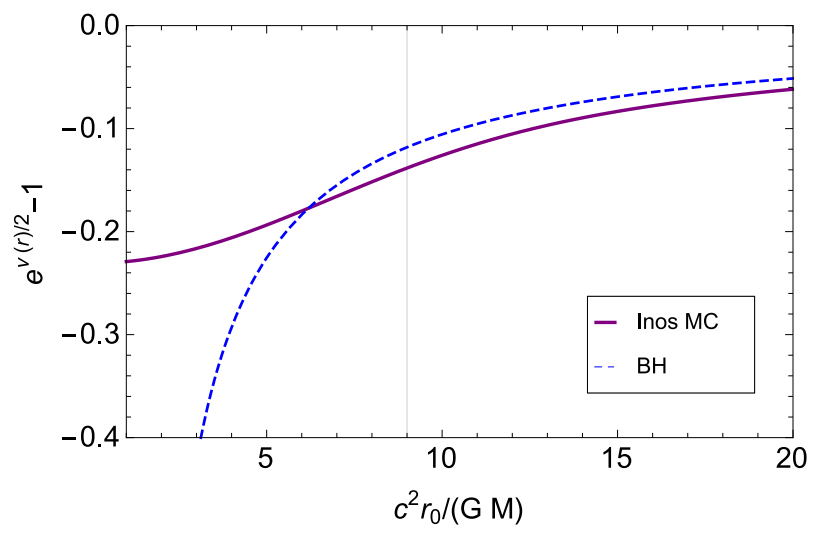

FIG. 7. Gravitational potential comparison between the central $\mathrm{BH}$ and the fermionic DM configuration. The vertical line indicates the core radius of the DMCC: $r_{c}=9 G M / c^{2}$.

pass through the DMCC since it does not possess an event horizon, implying a vanishing deflection angle as the light rays approach the very center of the configuration. This means that there is no photon sphere, neither inside nor outside the DMCC. This analysis leads to the expected result that the DMCC does not produce such strong bending effects as a central BH. Therefore, the computed deflection of light rays may be used to discriminate the different core compactness, if (highly accurate) observations of the light deflection are available on such short scales.

Such accurate measurements could be reached in the near future by the Event Horizon Telescope (EHT) project for the MW and for M $87^{6}$. It is also important to note that we only quantified the gravitational signal effect through the deflection of light, but a more robust study must be done, i.e. including the motion of stellar or gas components near the MW center, in order to figure out realistic features that may be discriminant from observations.

Finally, we compute in a general way the deflection angle both for a $\mathrm{BH}$ and for the DMCC along the entire galaxy in order to estimate the contribution of these compact objects in comparison to the DM halo, see Fig. 8. In both cases, we use the exact formula (21). Apart from a very small region near the center, the two solutions agree well up to reaching the regime where the contribution from DM halo cannot be neglected in comparison to the contribution from the $\mathrm{BH}$. Of course, the fermionic model has to be compared with a combination of black hole and a conventional DM model.

\footnotetext{
${ }^{6}$ http://www.eventhorizontelescope.org
}

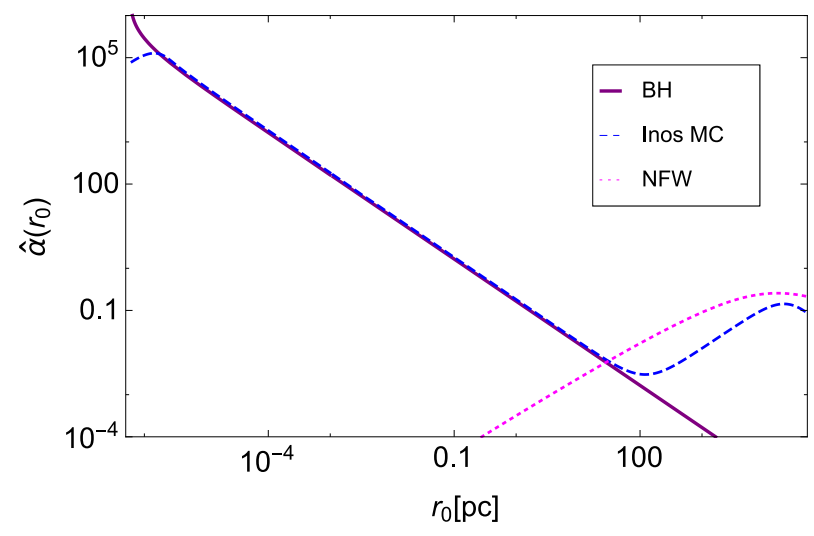

FIG. 8. Comparison between the BH lensing contribution along the entire galaxy as well as the most compact solution for the inos profile and the NFW profile. The deflection angle is given in arcsec.

\section{CONCLUDING REMARKS}

In this paper we studied for the first time the gravitational lensing properties of the fermionic DM distribution (RAR model) in galaxies [5, 7]. The RAR model describes correctly the properties of galactic DM halos of galaxies (including the flatness of the rotation curves) and, at the same time, it predicts a denser quantum core towards the center of the distribution. As it has been shown in Ref. [7], the compactness of the quantum core, for a fermion mass in the range $50 \mathrm{kev} \lesssim m c^{2} \lesssim 345 \mathrm{keV}$, is high enough to explain the dynamics of the S-cluster stars, the closest to the Galactic center. Thus, it represents an alternative scenario of the central compact object in Sgr $\mathrm{A}^{*}$, traditionally assumed to be a $\mathrm{BH}$.

We focused on the effect of the DM distribution in the lensing properties of hypothetical and nearby Milky Waylike spiral galaxies. We first studied the lensing effects caused by the DM halo region (i.e. neglecting the bulge and disk contributions to the net lensing). Then we performed the analysis of the strong bending features near the much denser lens represented by the DM quantum core at the galaxy center. We computed lensing properties such as convergence, deflection angle and magnification. We compared and contrasted the results for the RAR profile with the ones of phenomenological profiles such as the NFW and the NSIS ones.

For the fermion mass producing the most compact quantum DM core of the RAR profile (see Fig. 1), $m c^{2} \approx$ $345 \mathrm{keV}$, which can explain the Milky Way properties from the center all the way to the halo [7], we conclude:

1. At distances $r \gtrsim 20 \mathrm{pc}$ from the center of the galaxy, in the very inner DM halo regions where the diluted fermionic regime settles, the effect of the central object on the lensing properties, e.g. the deflection angle, is negligible (see, e.g., Figs. 4 and 8). 
2. Accurate measurements of the deflection angle in regions $r \gtrsim$ few kpc where DM starts to dominate, together with rotation curve data, could help to discriminate between different DM models (see Fig. 4).

3. The deflection angle at distances $r \lesssim 20 \mathrm{pc}$ from the center of the Galaxy increases in the case of the RAR model while it decreases for the phenomenological ones (see Figs. 4 and 8). The reason for this fundamental difference is the presence of the compact DM quantum core whose effects start to be appreciable below those distances (see next conclusions).

4. In the region of $10^{-6} \mathrm{pc} \lesssim r \lesssim 20 \mathrm{pc}$, the lensing effects of a quantum core and a central BH become a theme of unprecedented precision (see Fig. 6). The reason for this is that in this region the difference between the two cases is very small (see Fig. 7). This implies that the DM quantum core can affect lensing features such as the critical curves and the formation (suppression) of an additional (existing) faint central image in the same fashion as a central $\mathrm{BH}$.

5. The maximum deflection produced by the DM quantum core occurs at $\mathbf{r} \approx 7 G M_{c} / c^{2} \approx 1.4 \times$ $10^{-6}$ pc (see Fig. 6; $M_{c}$ is the mass of the DM core). This is produced with a characteristic demagnified signal as in the case of a central BH (see Fig. 5).

6. The effects of strong lensing (multiple images and Einstein rings) are important at short distances $10^{-4}$ pc for the more compact solution, when the condition $\kappa>1$ is achieved (see Fig. 3).

7. Large differences in the deflection angle produced by a DM central core and a central BH appear at distances $r \lesssim 10^{-6}$ pc (see Fig. 6). Inside this region the density of the DM quantum core is nearly constant (see Fig. 1) and its associated gravitational potential becomes weaker with respect to the one of a BH with the same mass (see Fig. 7). The reason is that the DMCC does not possess an event horizon, i.e it is regular at the center in contrast to the central BH, implying a vanishing deflection angle as the light rays approach the very center of the DMCC.

8. The quantum DM core does not show a photon sphere but it can generate multiple images and Einstein rings (see Fig. 5). Interestingly, the proposed Event Horizon Telescope uses a Very Long Baseline Interferometry (VLBI) array of (sub)millimeter telescopes that could resolve the predicted shadow of the central $\mathrm{BH}$ within the next years with the inclusion of the Atacama Large
Millimeter/submillimeter Array (ALMA). The expected angular resolution is 20-30 $\mu$ arcsec [41], whereas the predicted angular diameter of the shadow is $54 \mu$ arcsec. If a $\mathrm{BH}$ shadow will not be observed, then it will open a window for alternative scenarios regarding the nature of the SgrA* central object including the DM quantum core predicted by the RAR model.

Analogous conclusions apply as well to the RAR profiles obtained for other fermion masses in the range $50 \mathrm{kev} \lesssim m c^{2} \lesssim 345 \mathrm{keV}$ and for other galaxy types such as dwarf and elliptical galaxies, due to the universal behavior of the RAR density profiles (see Fig. 2 in [7]). The latter opens the interesting possibility to use the lensing data for single galaxies from surveys such as SWELLS [14, 15] and DiskMass [16].

We have considered in this work the gravitational lensing produced by fermionic DM distributions within the RAR model for isolated galaxies. Since the lensing is enhanced in clusters of galaxies, the generalization of the RAR profile in presence of galaxy interactions deserves to be explored in future works.

In Ref. [7] it has been shown that, for a fermion mass range $50 \mathrm{kev} \lesssim m c^{2} \lesssim 345 \mathrm{keV}$, the RAR profile is consistent both with the Milky Way data and, when applied to other galaxies, with observed galaxy correlations such as the $M_{\mathrm{BH}}-M_{\mathrm{DM}}$ relation and the constancy of the central surface DM density. Interestingly, in the case of a fermion mass of $m c^{2} \approx 50 \mathrm{keV}$, the DM core becomes gravitationally unstable to $\mathrm{BH}$ formation when it reaches a mass of $\approx 2.3 \times 10^{8} M_{\odot}$. This led to the hypothesis made in Ref. [7] that supermassive BHs $\left(M \gtrsim 10^{8} M_{\odot}\right)$ hosted at the center of active galaxies could be formed from a BH seed given by this DM collapse. Such a newly born $\mathrm{BH}$, soon after its formation, can accrete baryonic and DM from its surroundings. In that case, the fermionic DM density profile will be affected by the presence of, and accretion on to, the central $\mathrm{BH}$. We are planning to perform an analysis of the lensing properties of such a accretion-modified RAR profile, as well as its feedback on the BH shadow properties (see, e.g., Ref. [42], for the case of a NFW density profile) in a future publication.

\section{ACKNOWLEDGMENTS}

L.G.G. is supported by the Erasmus Mundus Joint Doctorate Program by Grant Number 2013-1471 from the EACEA of the European Commission. L.G.G is grateful for the warm hospitality at ZARM institute during his mobility period. C.R.A acknowledges support from ICRANet and CONICET-Argentina. J.A.R. acknowledges the support by the International Cooperation Program CAPES-ICRANet financed by CAPES Brazilian Federal Agency for Support and Evaluation of Graduate Education within the Ministry of Education of Brazil. 
[1] Planck Collaboration, P. A. R. Ade, N. Aghanim, M. Arnaud, M. Ashdown, J. Aumont, C. Baccigalupi, A. J. Banday, R. B. Barreiro, J. G. Bartlett, and et al., ArXiv e-prints (2015), arXiv:1502.01589.

[2] J. Binney and S. Tremaine, Galactic Dynamics: Second Edition, by James Binney and Scott Tremaine. ISBN 978-0-691-13026-2 (HB). Published by Princeton University Press, Princeton, NJ USA, 2008. (Princeton University Press, 2008).

[3] P.-H. Chavanis and J. Sommeria, MNRAS 296, 569 (1998).

[4] P.-H. Chavanis, Physica A Statistical Mechanics and its Applications 332, 89 (2004), cond-mat/0304073.

[5] R. Ruffini, C. R. Argüelles, and J. A. Rueda, MNRAS 451, 622 (2015), arXiv:1409.7365.

[6] I. Siutsou, C. R. Argüelles, and R. Ruffini, Astronomy Reports 59, 656 (2015), arXiv:1402.0695.

[7] C. R. Argüelles, J. A. Rueda, and R. Ruffini, ArXiv e-prints (2016), arXiv:1606.07040.

[8] G. P. Smith, J.-P. Kneib, H. Ebeling, O. Czoske, and I. Smail, ApJ 552, 493 (2001), astro-ph/0008315.

[9] J. Miralda-Escude, ApJ 370, 1 (1991).

[10] K. Umetsu, T. Broadhurst, A. Zitrin, E. Medezinski, and L.-Y. Hsu, ApJ 729, 127 (2011), arXiv:1011.3044 [astroph.CO].

[11] M. Meneghetti, M. Bartelmann, and L. Moscardini, MNRAS 340, 105 (2003), astro-ph/0201501.

[12] G. Hinshaw and L. M. Krauss, ApJ 320, 468 (1987).

[13] C. R. Keeton, ApJ 561, 46 (2001), astro-ph/0105200.

[14] A. A. Dutton, B. J. Brewer, P. J. Marshall, M. W. Auger, T. Treu, D. C. Koo, A. S. Bolton, B. P. Holden, and L. V. E. Koopmans, MNRAS 417, 1621 (2011), arXiv:1101.1622.

[15] M. Barnabè, A. A. Dutton, P. J. Marshall, M. W. Auger, B. J. Brewer, T. Treu, A. S. Bolton, D. C. Koo, and L. V. E. Koopmans, MNRAS 423, 1073 (2012), arXiv:1201.1692.

[16] T. P. K. Martinsson, M. A. W. Verheijen, K. B. Westfall, M. A. Bershady, D. R. Andersen, and R. A. Swaters, A\&A 557, A131 (2013), arXiv:1308.0336.

[17] C. R. Keeton and C. S. Kochanek, ApJ 495, 157 (1998), astro-ph/9705194.

[18] S. Courteau and A. A. Dutton, ApJ 801, L20 (2015), arXiv:1502.04709.

[19] C. Grillo, ApJ 747, L15 (2012), arXiv:1202.3791 [astroph.CO].

[20] A. Sonnenfeld, T. Treu, R. Gavazzi, P. J. Marshall, M. W. Auger, S. H. Suyu, L. V. E. Koopmans, and A. S. Bolton, ApJ 752, 163 (2012), arXiv:1111.4215.

[21] C. Tortora, F. La Barbera, N. R. Napolitano, A. J. Romanowsky, I. Ferreras, and R. R. de Carvalho, MNRAS 445, 115 (2014), arXiv:1409.0538.

[22] Y. D. Hezaveh, P. J. Marshall, and R. D. Blandford, ApJ 799, L22 (2015), arXiv:1501.01757.
[23] M. I. N. Kobayashi, A. Leauthaud, S. More, N. Okabe, C. Laigle, J. Rhodes, and T. T. Takeuchi, MNRAS 449, 2128 (2015), arXiv:1502.06604.

[24] S. Mao, H. J. Witt, and L. V. E. Koopmans, MNRAS 323, 301 (2001), astro-ph/0007011.

[25] J. N. Winn, D. Rusin, and C. S. Kochanek, Nature 427, 613 (2004), astro-ph/0312136.

[26] K. S. Virbhadra and G. F. R. Ellis, Phys. Rev. D 62, 084003 (2000), astro-ph/9904193.

[27] J. F. Navarro, C. S. Frenk, and S. D. M. White, ApJ 462, 563 (1996), astro-ph/9508025.

[28] P. R. Shapiro, I. T. Iliev, and A. C. Raga, MNRAS 307, 203 (1999), astro-ph/9810164.

[29] S. Gillessen, F. Eisenhauer, T. K. Fritz, H. Bartko, K. Dodds-Eden, O. Pfuhl, T. Ott, and R. Genzel, ApJ 707, L114 (2009), arXiv:0910.3069.

[30] Y. Sofue, PASJ 65 (2013), 10.1093/pasj/65.6.118, arXiv:1307.8241.

[31] Y. Sofue, M. Honma, and T. Omodaka, PASJ 61, 227 (2009), arXiv:0811.0859.

[32] P. Schneider, J. Ehlers, and E. E. Falco, Gravitational Lenses (Springer-Verlag Berlin Heidelberg New York, 1992) p. 230.

[33] E. M. Shin and N. W. Evans, MNRAS 374, 1427 (2007), astro-ph/0611134.

[34] S. Bharadwaj and S. Kar, Phys. Rev. D 68, 023516 (2003), astro-ph/0304504.

[35] T. Faber and M. Visser, MNRAS 372, 136 (2006), astro$\mathrm{ph} / 0512213$.

[36] D. Núñez, A. X. González-Morales, J. L. CervantesCota, and T. Matos, Phys. Rev. D 82, 024025 (2010), arXiv: 1006.4875 .

[37] S. Weinberg, Gravitation and Cosmology: Principles and Applications of the General Theory of Relativity (WileyVCH, 1972) p. 688.

[38] N. Bilić, H. Nikolić, and R. D. Viollier, ApJ 537, 909 (2000), astro-ph/9912381.

[39] M. P. Dabrowski and F. E. Schunck, ApJ 535, 316 (2000), astro-ph/9807039.

[40] V. Perlick, Living Rev. Relativity 7(9) (2004), http://www.livingreviews.org/lrr-2004-9.

[41] S. Doeleman, E. Agol, D. Backer, F. Baganoff, G. C. Bower, A. Broderick, A. Fabian, V. Fish, C. Gammie, P. Ho, M. Honman, T. Krichbaum, A. Loeb, D. Marrone, M. Reid, A. Rogers, I. Shapiro, P. Strittmatter, R. Tilanus, J. Weintroub, A. Whitney, M. Wright, and L. Ziurys, in astro2010: The Astronomy and Astrophysics Decadal Survey, Astronomy, Vol. 2010 (2009) arXiv:0906.3899 [astro-ph.CO].

[42] T. Lacroix and J. Silk, A\&A 554, A36 (2013), arXiv:1211.4861 [astro-ph.GA] . 